Int. J. Gynaecol. Obstet., 1982, 20: 417-423

International Federation of Gynaecology \& Obstetrics

\title{
CHEMOTHERAPY IN ADVANCED PRIMARY AND RECURRENT CERVICAL CARCINOMA
}

\section{YEW CHEONG CHOO}

Division of Gynecologic Oncology, Department of Obstetrics and Gynecology, University of Michigan Medical School, Ann Arbor, $M I, U S A$

(Received May 11th, 1981)

(Accepted April 13th, 1982)

\begin{abstract}
Choo YC (Division of Gynecologic Oncology, Department of Obstetrics and Gynecology, University of Michigan Medical School, Ann Arbor, MI, U.S.A.). Chemotherapy in advanced primary and recurrent cervical carcinoma.

Int J Gynaecol Obstet 20: 417-423, 1982

Chemotherapy in carcinoma of the cervix continues to be a therapeutic challenge. The diversified treatment programs from one institution and the factors affecting response are analyzed. It is concluded that until more active regimens are available, chemotherapy in advanced and recurrent cervical carcinoma should be confined to patients with good prognostic factors.
\end{abstract}

Key words: Cervical neoplasm; Drug therapy

\section{Introduction}

The incidence of invasive carcinoma of the cervix has declined considerably within the past decade due largely to the detection and treatment of the pre-invasive disease, but stage for stage, no improvement in the survival of the invasive disease has been recorded. While surgery and radiation are equally efficacious for the early stages and radiation only is employed for the later stages, chemotherapy plays a role in metastatic primary disease and advanced recurrent disease. Adjunctive chemotherapy has been suggested in patients in whom paraaortic nodal metastasis have occurred when the disease is considered systemic, but its value is unknown. The response and success of chemotherapeutic agents in carcinoma of the cervix have been disappointing; the overall response rate is generally around $20-30 \%$, usually lasting for a few months only, and complete remission is rare [1]. In recent years, one witnesses the evolution of single to multiple agents in the chemotherapy of carcinoma of the cervix. Drugs that have been reported to be active are as shown in Tables I and II. At the University of Michigan Hospital, diversified regimens of chemotherapy have been employed in keeping with this trend; these also provided valuable experience in the use of various drugs. This study serves to analyze our results in the application of these agents

Table I. Single agents active in carcinoma of the cervix.

Cyclophosphamide
5-Fluorouracil
Chlorambucil
Melphalan
Methotrexate
Adriamycin
Bleomycin
Me CCNU
Hexamethylmelamine
Mitomycin C
Profibromycin
Cis-platinum

Cyclophosphamide

5-Fluorouracil

mbucil

Melphalan

Methotrexate

Me CCNU

Hexamethylmelamine

Cis-platinum 
Table II. Combination agents active in carcinoma of the cervix.

Bleomycin/mitomycin C [2]

Vincristine/bleomycin/mitomycin C [3]

Adriamycin/methotrexate [4]

Doxorubicin/methyl CCNU [5]

Adriamycin/cy toxan [6]

Bleomycin/methotrexate [7]

Adriamycin/cis-platinum [8]

Adriamycin/cytoxan/5-fluorouracil [9]

Bleomycin/methotrexate/cis-platinum [10]

and to categorize prognostic factors, if any, related to patient selection and response in chemotherapy treatment of carcinoma of cervix.

\section{Materials and methods}

During the 6-year period from January 1974 through December 1979, 523 cases of cervical carcinoma were seen at this institution. Nineteen patients had metastasis outside the pelvis when first seen (Stage IVB), or developed metastasis during or soon after radiotherapy. Eighty patients developed advanced recurrent carcinoma following initial surgery or radiation. Chemotherapy would have formed the basis of treatment in these 99 patients but 19 patients either refused chemotherapy or had such a low Kanofsky index that chemotherapy would not deem to be beneficial. At this institution, the gynecologic oncologist is primarily responsible for chemotherapy of gynecologic cancers. Single agents that have been used in this institution in cancer of the cervix included cytoxan,

Table III. Single agents used in carcinoma of the cervix at the University of Michigan Hospital.

Cytoxan, $100 \mathrm{mg} / \mathrm{m}^{2}$ p.o. or $1 \mathrm{~g} / \mathrm{m}^{2}$ i.v. $\mathrm{q} 3$ weeks.

Methotrexate, $25 \mathrm{mg}$ i.v. weekly.

Adriamycin, $60 \mathrm{mg}$ i.v. q $3-4$ weeks.

Cis-platinum, $60-100 \mathrm{mg} / \mathrm{m}^{2}$ i.v. $\mathrm{q} 3$ weeks.

Bleomycin infusion, $20 \mathrm{mg} / \mathrm{day} \times 5$ days $\mathrm{q} 4$ weeks. methotrexate, adriamycin, cis-platinum and bleomycin infusion (Table III). Combination agents used included adriamycin/cytoxan, bleomycin/vincristine/ mitomycin $\mathrm{C} /$ methotrexate (BOMM), BOMM induction followed by consolidation treatment with adriamycin/cis-platinum/cytoxan (BOMM-APC), vincristine/5-fluorouracil infusion, vincristine/mitomycin $\mathrm{C} /$ bleomycin (Table IV). The BOMM and BOMM-APC regimens were adopted from protocols used by the Medical Oncology Department for head and neck cancers. Adriamycin/cytoxan regimen was the most common combination used, but lately BOMM-APC regimen was given more frequently. All patients underwent appropriate work-up studies which included an IVP, cystoscopy, sigmoidoscopy, chest X-ray, biochemical blood tests, and a liver and bone scan when indicated. Most of the patients also had a lymphangiogram, and selected patients also underwent fine needle aspiration of the abnormal nodes under fluoroscopy for cytology when their lymphangiograms were abnormal. The standard criteria for response were used. Complete response was defined as complete absence of detectable disease for 3 months or more; partial response as $50 \%$ or more reduction in the product of the perpendicular measurable diameters for 3 months or more. Lesser degree of responsiveness was classified as stable disease. Progression was defined as increase in size of the measurable lesion. In a few instances where the lesion was not clinically evident such as disease proven in the lymph nodes, the disease free interval was used as the criterion of response.

\section{Results}

Primary carcinoma - persistent or metastatic

Table V lists the 19 patients with primary carcinoma of the cervix and the indications for chemotherapy. Twelve patients had Stage IVB disease while the rest had metastasis during or soon after radiation, or had persistent disease not amenable to surgery. 
Table IV. Combination chemotherapy used in cervical cancer at the University of Michigan Hospital.

1. Adriamycin/cytoxan Adriamycin, $60 \mathrm{mg} / \mathrm{m}^{2}$ i.v. $\mathrm{q} 3$ weeks Cytoxan, $600 \mathrm{mg} / \mathrm{m}^{2}$ i.v. $\mathrm{q} 3$ weeks

2. Bleomy cin/vincristine/mitomycin $\mathrm{C} /$ methotrexate (BOMM)

Vincristine, $1.5 \mathrm{mg} / \mathrm{m}^{2}$ i.v., repeat in 4 weeks

Mitomycin, $\mathrm{C}, 15 \mathrm{mg} / \mathrm{m}^{2} 6 \mathrm{~h}$ after vincris tine at initial course only

Bleomycin, $20 \mathrm{U} / 24 \mathrm{~h}$ infusion $\times 4$ days. Repeat in 4 weeks.

Methotrexate, $30 \mathrm{mg} / \mathrm{m}^{2}$ i.v. $36 \mathrm{~h}$ after bleomycin infusion, then $\mathrm{q} 1$ week

3. Vincristine/bleomycin/mitomycin $C$. As in BOMM but without methotrexate

4. Blcomycin/vincristine/mitomycin C/methotrexate-Adriamycin/Cis-platinum/cytoxan (BOMM-APC)

Vincristine, $2.0 \mathrm{mg}$ i.v. $\mathrm{q} 4$ weeks $\times 2,6 \mathrm{~h}$ prior to mitomycin $\mathrm{C}$ and bleomycin infusion

Mitomycin $C, 15 \mathrm{mg} / \mathrm{m}^{2}$ i.v. at beginning of first bleomycin infusion only

Bleomycin, 20 units $/ 24 \mathrm{~h} \times 4$ days $\mathrm{q} 4$ weeks $\times 2$

Methotrexate, $30 \mathrm{mg} / \mathrm{m}^{2}$ i.v. $36 \mathrm{~h}$ after bleomycin, then twice weekly

Leukovorin factor, $20 \mathrm{mg} 24 \mathrm{~h}$ after methotrexate

Cis-platinum, $60 \mathrm{mg} / \mathrm{m}^{2}$ infusion 4 weeks after BOMM and $\mathrm{q} 3$ weeks

Adriamycin, $40 \mathrm{mg} / \mathrm{m}^{2}$ i.v. 4 weeks after BOMM and $\mathrm{q} 3$ weeks

Cytoxan, $400 \mathrm{mg} / \mathrm{m}^{2}$ i.v. 4 weeks after BOMM and q 3 weeks

5. Vincris tine/5-fluorouracil

Vincristine, $1.5 \mathrm{mg} / \mathrm{m}^{2}$ i.v. days 1 and $2 \mathrm{q} 4$ weeks

5-Fluorouracil, $600 \mathrm{mg} / \mathrm{m}^{2} / 24 \mathrm{~h} \times 5$ days q 4 weeks

The sites of disease or metastasis are as shown in Table VI. In their initial work up, six had unilateral ureteral obstruction; 10 had an abnormal lymphangiogram for which five lymph node aspirations were performed, yielding three positive cytology. Radiation treatment was instituted in 15 patients seven had palliative cesium. The remaining four patients had metastasis outside the pelvis but minimal local symptoms and they received chemotherapy only. Of the 15 patients who were given radiation, 12 also received adjunctive chemotherapy while three either refused or were unfit for chemotherapy.

The first line chemotherapy and the response are shown in Table VII. Complete response was noted in three patients (18.8\%), partial response in one $(6.3 \%)$, and stable disease in two $(12.5 \%)$. Three out of eight patients who received adriamycin/cytoxan regimen responded $(37.5 \%)$ : one patient who had unilateral ureteral obstruction and lymph node metastasis in the neck treated by radical radiation and chemotherapy is alive with no disease for 5 years; one patient had partial response for 7 months but succumbed to progressive disease 3 months later; another patient who had multiple metastasis to the lungs but minimal cervical disease had partial response and is alive after 20 months of treatment with adriamycin/cytoxan regimen only. Cytoxan resulted in complete remission in

Table V. Chemotherapy in advanced primary carcinoma of the cervix - stages and indications.

\begin{tabular}{|c|c|c|}
\hline Stage & No. & Indication \\
\hline IB & 1 & Metastasis to lungs while on radiation \\
\hline II & 1 & Lymph node metastasis to the neck on completion of radiation \\
\hline III & 2 & Persistent disease following radiation \\
\hline IVA & 2 & 1 , persistent disease following radiation and the other, metastasis to the spine while on radiation \\
\hline IVB & 12 & Metastasis outside the pelvis \\
\hline Unstaged & 1 & Inadvertent hysterectomy followed by radiation. Persistent disease \\
\hline
\end{tabular}


Table VI. Sites of metastasis in patients with primary carcinoma of the cervix.

\begin{tabular}{ll}
\hline Lung & 7 \\
Neck nodes & 6 \\
Pelvic and/or paraa ortic nodes & 4 \\
Spine & 1 \\
Spine and lungs & 1 \\
& 19 \\
\hline
\end{tabular}

a patient who had adenocarcinoma of the cervix with metastasis to the lungs for 20 months but she died of a carcinoma of the breast. Two patients on BOMM-APC are presently alive: one has stable disease for 6 months and the other has complete response for 9 months. When these patients are analyzed, it is observed that two patients were given radical radiation, two had palliative cesium, and in the other two, no radiation at all was given. The sites of metastasis were the supraclavicular nodes in five and the lungs in one. Also noted was the absence of clinical local disease following treatment.

Second line chemotherapy was instituted in five patients but none showed any response.

Among those patients who did not respond to chemotherapy, the average survival was 5.9 months. The three patients who received no chemotherapy but only palliative cesium treatment died of their disease after an average interval of 6 months.
Complications of chemotherapy were noted in only four patients: three had mild to moderate leukopenia, one had severe leukopenia and sepsis from BOMM-APC and required antibiotics, one patient had reduced EKG voltages necessitating discontinuation of adriamycin.

\section{Recurrent carcinoma}

Among the 80 patients who had recurrent cervical carcinoma, three had initial radical hysterectomy, 69 were treated by radiation for their primary disease and eight had radical hysterectomy or inadvertent simple hysterectomy followed by radiation. Their original disease was Stage I in 17, Stage II in 38, Stage III in six, Stage IV in three, and unstaged or unknown in 16 patients. The sites of recurrence were pelvic, unamenable to exenteration in 47; distant metastasis with no pelvic recurrence in 22 , and both distant metastasis and pelvic recurrence in 11 patients.

Thirty-nine patients who had pelvic recurrence had lymphangiographic study performed, 32 were reported as abnormal. Lymph node aspiration was performed on 11 patients, yielding seven positive cytology. Seventeen patients had positive direct lymph node biopsies, this was performed for exploration for possible exenteration or to ascertain a negative lymph node aspiration. During the initial period, patients who had ureteral obstruction as evidenced on IVP were invariably explored but all were discovered to have nodal spread of disease as well as

Table VII. First line chemotherapy and response in patients with advanced primary carcinoma of the cervix.

\begin{tabular}{|c|c|c|c|c|c|c|}
\hline \multirow[t]{2}{*}{ Regimen } & \multicolumn{6}{|c|}{ Response } \\
\hline & Stable & Partial & Complete & Progression & Total & \\
\hline Adriamycin/cytoxan & & 2 & 1 & 5 & 8 & \\
\hline Cytoxan & & & 1 & 3 & 4 & . \\
\hline BOMM-APC & 1 & & 1 & & 2 & \\
\hline Vincristine/5-fluorouracil & & & & 1 & 1 & \\
\hline Cis-platinum & & & & 1 & 1 & \\
\hline TOTAL & & & & & 16 & \\
\hline
\end{tabular}


Table VIII. First line chemotherapy and response in patients with recurrent carcinoma of the cervix.

\begin{tabular}{|c|c|c|c|c|c|}
\hline \multirow[t]{2}{*}{ Regimen } & \multicolumn{5}{|c|}{ Response } \\
\hline & Stable & Partial & Complete & Progression & Total \\
\hline Adriamycin/cytoxan & 7 & & & 19 & 26 \\
\hline Adriamycin & 2 & & & 13 & 15 \\
\hline Cytoxan & 2 & & & 7 & 9 \\
\hline BOMM & 2 & & & 3 & 5 \\
\hline BOMM-APC & 1 & & & & 1 \\
\hline Cis-platinum & 2 & & & 2 & 4 \\
\hline Vincristine/5-fluorouracil & 1 & & & 1 & 2 \\
\hline Bleomycin & & & & 1 & 1 \\
\hline \multicolumn{6}{|l|}{ Vincristine/mitomycin C/ } \\
\hline bleomycin & & & & 1 & 1 \\
\hline TOTAL & 17 & & & 47 & 64 \\
\hline
\end{tabular}

disease spread to pelvic side wall. More recently, lymphangiograms were performed on these patients revealing a high rate of suspicious nodes. Such patients would then undergo fine needle aspiration of the suspicious nodes and with this technique we have obviated many unnecessary explorations.

Sixty-four patients received first line chemotherapy as shown in Table VIII. None had partial or complete remission. Fourteen patients had stable disease or disease free interval for an average duration of 7.7 months $(21.9 \%)$, and for these patients, the average survival was 19.6 months. On the other hand, for those who failed to respond, the average survival was 7.6 months. For the remaining 16 patients who received no chemotherapy, their average survival was 7.5 months.

Fifteen patients received second line chemotherapy, one partial response was recorded with BOMM for 3 months, unfortunately she succumbed to pulmonary toxicity. Two patients given cytoxan treatment had stable disease for an average duration of 9 months.

Third line chemotherapy was instituted in seven patients, none had any response.

Adriamycin/cytoxan was the most common regimen in first line treatment, and stable disease was recorded in seven out of 26 patients $(26.9 \%)$.

Mild to moderate leukopenia occurred in eight patients. One patient had severe leukopenia requiring prophylactic antibiotics. Two patients died from bleomycin pulmonary toxicity.

Analyzing the response to chemotherapy in relation to the location of the recurrent disease, it is noted that among 15 patients who had stable disease on first line chemotherapy, 12 had distant recurrences without pelvic disease. Three response was observed in patients who had recurrence in the pelvis. All three patients who responded to second line chemotherapy again had disease in distant sites and not the pelvis.

The stage of the initial disease and the interval between initial treatment and recurrence had no bearing on their response to chemotherapy.

Ureteral obstruction was an ominous sign for prognosis in this group of patients. With unilateral obstruction, none lived for more than 8 months despite initial response from chemotherapy. With the presence of triad, i.e. unilateral ureteral obstruction, unilateral leg edema and leg pain, the survival dropped further to an average of 5.7 months. Iliofemoral venous obstruction also signified poor prognosis, and in two patients, their survival was less than 6 months.

\section{Discussion}

Despite many diversified chemotherapeutic 
agents being employed and new drug combinations being reported, carcinoma of the cervix is still regarded a chemo-resistant cancer. The often mentioned difficulties encountered in chemotherapy in cervical cancer are: decreased chemo-perfusion when recurrence occurs in a previously irradiated pelvis; renal function impairment from ureteral obstruction precluding the use of active agents such as methotrexate and cisplatinum and affecting the pharmokinetics of many other agents; difficulty in evaluating responses because of prior fibrosis in the pelvis, and compromised bone marrow from prior irradiation of some one-third functional bone marrow affecting aggressive drug treatment.

This study does not aim to compare the response and results of different chemotherapy agents but serves to emphasize that chemotherapy can play a role in selected patients with primary metastatic and recurrent carcinoma of the cervix.

Since only a small percentage of patients with cervical cancer requiring chemotherapy will respond, it is vital to know who the potential candidates are and what kind of active agents are available. Also, the benefits of drug treatment should balance against potential complications and fatalities from such treatment. Cumulative reports have shown that active drugs presently employed in cancer of the cervix are confined mainly to adriamycin, cytoxan, methotrexate, cis-platinum, vincristine, bleomycin and mitomycin $\mathrm{C}$. Although multiple combination agents may show enhanced response, their effectiveness has not been proven to be superior to single agent alone; besides, the potential bone marrow depression warrants caution in their use. Drugs with irreversible potential complications such as bleomycin and cis-platinum should be used with extreme care with close monitoring. In our experience, adriamycin/cytoxan combination is an active regimen despite report to its contrary [1.1]. Its acceptability and practicality also lie in its ease of administration with bolus injections at 3-4 weekly intervals without hospitalization, and its rarity of severe complications. The BOMM-APC regimen does show promise and further evaluation is underway.

Favorable prognostic factors are distant metastasis to the lungs and lymph nodes; in particular patients with such metastasis without pelvic recurrence are favorable candidates for chemotherapy. The presence of ureteral obstruction alone, ilio-femoral vein thrombosis, and the triad of abnormal IVP, unilateral leg pain and edema are deterrans precluding favorable response to chemotherapy.

This study shows that partial and complete remissions are more frequent with primary metastatic carcinoma than recurrent carcinoma. The poorer result in the latter may be related to impaired vascular perfusion in the pelvis. But when patients with primary metastatic carcinoma fail to respond to chemotherapy, their course is more rapid than those with recurrent disease.

Regardless of the type of disease, however, for those who respond to chemotherapy, their survival can be meaningfully improved as opposed to those who do not respond. Those who fail to respond to first line chemotherapy in all likelihood will probably not respond to further chemotherapy.

\section{References}

1 Wasserman TH, Carter SK: The intergration of chemotherapy in to the combined modality treatment of solid tumors. VIII Cervical cancer. Cancer Treat Rev 4: 25, 1977.

2 Miyamoto T, Takabe $Y$, Watanabe M, Terasima T: Effectiveness of a sequential combination of bleomycin and mitomycin $C$ in advanced cervical cancer. Cancer 41 : 403, 1978 .

3 Baker LH, Opipari MI, Wilson H, Bottomly R, Coltman $\mathrm{CA}$ : Mitomycin $\mathrm{C}$, vincristine and bleomycin therapy for advanced cervical cancer. Obstet Gynecol 52: 146, 1978.

4 Guthrie D, Way S: Treatment of advanced carcinoma of the cervix with adriamycin and methotrexate combined. Obstet Gynecol 44: 586, 1974.

5 Day TG, Wharton JT, Gottlieb JA, Rutledge FN: Chemotherapy for squamous carcinoma of the cervix: Doxo- 
rubicin-methyl CCNU. Am J Obstet Gynecol 132: 545, 1978.

6 Alberto DS, Ignoffo R: Adriamycin-cyclophosphamide treatment of squamous carcinoma of the cervix. Cancer Treat Rep 62: 143, 1978.

7 Conroy JF, Lewis GC, Brady LW, Brodsky I, Kahn S, Ross D, Nuss R: Low dose bleomycin and methotrexate in cervical cancer. Cancer 37: 660, 1976.

8 Slayton RE, Mladineo JP: Adriamycin and cis-diamminedichloroplatinum (DDP) in recurrent and metastatic squamous carcinoma of the cervix. A pilot study. Proc Am Assoc Cancer Res 19: 335, Abstract No. C-114, 1978.

9 Piver MS, Barlow JJ, Xynos FP: Adriamycin alone or in combination in 100 patients with carcinoma of the cervix or vagina. Am J Obstet Gynecol 131: 311, 1978.

10 Vogl SE, Moukhtar M, Kaplan BH: Chemotherapy for advanced cervical cancer with methotrexate, bleomycin and cis-dichlorodiammineplatinum. Cancer Treat Rep 63: $1005,1979$.

11 Wallace HJ Jr, Hreschyshyn MM, Wilkank GD, Boronow RC, Fowler WC Jr, Blessing JA: Comparison of the therapeutic effects of adriamycin alone versus adriamycin plus vincristine versus adriamycin plus cyclophosphamide in the treatment of advanced carcinoma of the cervix. Cancer Treat Rep 62: 1435, 1978.

\section{Address for reprints:}

Dr. Y.C. Choo

Department of Obstetrics and Gynaecology

Queen Mary Hospital

University of Hong Kong

Hong Kong 